\title{
EZH2 protein: a promising immunomarker for the detection of hepatocellular carcinomas in liver needle biopsies
}

\author{
Mu-Yan Cai, ${ }^{1,2}$ Zhu-Ting Tong, ${ }^{1}$ Fang Zheng, ${ }^{1}$ Yi-Ji Liao, ${ }^{1}$ Yi Wang, ${ }^{1}$ Hui-Lan Rao, ${ }^{1,2}$ \\ Yang-Chao Chen, ${ }^{3}$ Qiu-Liang Wu ${ }^{1,2}$ Yan-Hui Liu, ${ }^{4}$ Xin-Yuan Guan, ${ }^{1}$ Marie C Lin, ${ }^{1,3}$ \\ Yi-Xin Zeng, ${ }^{1}$ Hsiang-Fu Kung, ${ }^{1,3}$ Dan $\mathrm{Xie}^{1}$
}

\begin{abstract}
See Commentary, p 881
${ }^{1}$ State Key Laboratory of Oncology in South China, Cancer Center, Sun Yat-Sen University, Guangzhou, China ${ }^{2}$ Department of Pathology, Cancer Center, Sun Yat-Sen University, Guangzhou, China ${ }^{3}$ State Key Laboratory of Oncology in South China, Chinese University of Hong Kong, Hong Kong, China ${ }^{4}$ Department of Pathology and Laboratory Medicine, Guangdong Provincial People's Hospital, Guangzhou, Guangdong, China
\end{abstract}

\section{Correspondence to}

Dr Dan Xie, State key Laboratory of Oncology in South China, Cancer Center, Sun Yat-Sen University, No. 651, Dongfeng Road East, 510060 Guangzhou, China; xied@mail.sysu.edu.cn

Revised 19 January 2011 Accepted 20 January 2011 Published Online First 17 February 2011

\section{ABSTRACT}

Background and aims $A$ previous study of ours indicated that enhancer of zeste homologue 2 (EZH2) plays an important role in hepatocellular carcinoma (HCC) tumorigenesis. The aim of the present study was to investigate the potential diagnostic utility of EZH2 in HCC.

Methods Immunohistochemistry was performed to examine the expression dynamics of $\mathrm{EZH} 2$ in two independent surgical cohorts of HCC and non-malignant liver tissues to develop a diagnostic yield of EZH2, HSP70 and GPC3 for HCC detection. The diagnostic performances of EZH2 and a three-marker panel in HCC were re-evaluated by using an additional biopsy cohort. Results Immunohistochemistry analysis demonstrated that the sensitivity and specificity of EZH2 for HCC detection was $95.8 \%$ and $97.8 \%$ in the testing cohort. Similar results were confirmed in the validation cohort. For diagnosis of well-differentiated HCCs, the sensitivity and specificity were $68.9 \%$ and $91.5 \%$ for EZH2, $62.5 \%$ and $98.5 \%$ for HSP70, $50.0 \%$ and $92.1 \%$ for GPC3, and $75.0 \%$ and $100 \%$ for a three-marker panel. In biopsies, positive cases for at least one marker increased from large regenerative nodule and hepatocellular adenoma (0/12) to focal nodular hyperplasia (2/20), dysplastic nodule (7/25), well-differentiated HCC (16/18) and moderately and poorly differentiated HCC (54/54). When at least two positive markers were considered, regardless of their identity, the positive cases were detected in 0/12 large regenerative nodules and hepatocellular adenomas, 0/20 focal nodular hyperplasias, 0/25 dysplastic nodules, 11/18 welldifferentiated HCCs, 32/37 moderately differentiated HCCs and 15/17 poorly differentiated HCCs.

Conclusion Our findings suggest that EZH2 protein, as examined by immunohistochemistry, may serve as a promising diagnostic biomarker of HCCs, and the use of a three-marker panel (EZH2, HSP70 and GPC3) can improve the rate of detection of HCCs in liver biopsy tissues.

\section{INTRODUCTION}

Hepatocellular carcinoma (HCC) is a major lethal malignancy. The disease has a high prevalence in southeast Asia and Africa, and the incidence of HCC has also been steadily increasing in Europe and America. ${ }^{1} 2$ Due to the high prevalence of hepatitis $B$ virus (HBV) infection in Chinese populations, HBV-related liver cirrhosis and/or

\section{Significance of this study}

What is already known about this subject?

- Needle biopsy is recommended to identify the features of liver malignancies when small hepatic nodules are detected. Clinically, it is difficult to make a histological distinction between well-differentiated hepatocellular carcinomas (WD HCCs) and dysplastic nodules (DNs), especially in liver biopsy tissues. EZH2 plays a key role in the tumorigenesis of HCC. Increased expression of EZH2 has been frequently detected in HCC tissues, and has been correlated with the aggressiveness and poor prognosis of HCCs.

\section{What are the new findings?}

- The staining intensity of EZH2 by immunohistochemistry in WD HCCs is significantly larger than that in DNs.

- The evaluation of EZH2 expression enables us not only to discriminate HCCs and non-neoplastic liver tissue, but also to distinguish WD HCC from DNs with a high degree of accuracy.

- The combination of the three markers (EZH2, HSP70 and GPC3) could greatly improve our ability to diagnose HCCs with better sensitivity and optimal specificity.

\section{How might it impact on clinical practice in the foreseeable future? \\ - The results reported here suggest that EZH2 can serve as a promising immunomarker of HCCs in the diagnostic work-up of the liver lesions, and that the use of a three-marker panel (EZH2, HSP70 and GPC3) enables HCCs to be detected more easily in liver biopsies.}

HCC has become one of the main disease burdens in China. Unfortunately, the long-term prognosis of patients with HCC remains unsatisfactory in spite of recent advances in surgical techniques and medical management. ${ }^{3}$ This poor outcome is, in part, attributable to the fact that HCC is generally diagnosed at a late clinical stage, when treatments are of limited effectiveness. ${ }^{4}$ Although serum $\alpha$-fetoprotein (AFP) and ultrasonography are routinely used in diagnosis at present, 
complementing clinical assessments, they have a relatively low sensitivity and specificity, and are not able to identify small and early stage HCCs. ${ }^{5}$ To diagnose small and early stage HCCs clinically, a biopsy of hepatic nodules is usually recommended as a check on diagnoses deriving from serological and radiological tests. ${ }^{4}$ However, it is difficult to make a histological distinction between early well-differentiated (WD) HCC and certain benign hepatocellular lesions, such as dysplastic nodule (DN) in liver biopsy specimens, even for experienced pathologists; while immunomarkers commonly used by clinicians, such as AFP, CD34 and carcinoembryonic antigen (CEA), have significant diagnostic limitations. ${ }^{7-9}$ There is therefore an urgent need to discover and identify new biomarkers that can distinguish between HCCs and other benign liver lesions in liver needle biopsy tissues.

Enhancer of zeste homologue 2 (EZH2), the catalytic subunit of polycomb repressive complex 2 (PRC2), has been identified as the sole histone methyltransferase that methylates histone $\mathrm{H} 3$ lysine 27 (H3K27) and mediates transcriptional silencing. ${ }^{10}$ $\mathrm{EZH} 2$ has been found to contribute to the maintenance of cell identity, cell cycle regulation and oncogenesis. ${ }^{11}{ }^{12}$ A previous study by our group showed that knockdown of EZH2 expression in HCC cells was sufficient to significantly reverse tumorigenicity in a nude mouse model, and demonstrated the potential therapeutic value of EZH2 inhibition in HCC. ${ }^{13}$ Recent studies have reported that increased expression of EZH2 was frequently detected in HCC tissues and it was correlated with the aggressiveness and/or poor prognosis of HCCs. ${ }^{14-16}$ However, these results were evaluated from a small cohort of liver tissue samples, and the criteria for evaluation of EZH2 positive expression were ambiguous. The potential utility of EZH2 as a diagnostic biomarker of HCC in liver resection and needle biopsy tissues still remains to be demonstrated.

The aim of the present study was to investigate the potential diagnostic utility of EZH2 in HCCs. We first analysed the protein levels of EZH2 in a panel of HCC and hepatic cell lines and tissues by western blotting. We then performed immunohistochemistry (IHC) to examine the expression pattern of EZH2 in a large surgical cohort of HCCs and corresponding non-neoplastic liver tissues. Two other potential molecules, heat-shock protein 70 (HSP70) and glypican 3 (GPC3), have previously been proposed for use in the differential diagnosis of hepatocallular nodules. These molecules provided a predictive power for the diagnosis of early HCC, ${ }^{17-20}$ and have been recently identified as two useful biomarkers in the diagnosis of HCC. ${ }^{21} 22$ We therefore further validated the diagnostic value of $\mathrm{EZH} 2$ both alone and in combination with HSP70 and GPC3 by IHC in another independent surgical cohort of HCCs and benign hepatic lesions. Finally, we reevaluated the diagnostic performances of EZH2, together with HSP70 and GPC3, in HCCs by using an additional cohort of liver needle biopsy samples. We now report for the first time that the expression of EZH2 protein, as examined by IHC, may serve as a promising diagnostic biomarker of HCC, and that the use of EZH2 in combination with HSP70 and GPC3 improves the rate of detection of HCCs in liver needle biopsy tissues.

\section{MATERIALS AND METHODS \\ Cell line and cell cultures}

Six HCC cell lines (Huh7, HepG2, 7402, 7721, Hep3B and Lm3) and one normal hepatic cell line (Lo2) were maintained in Dulbecco's modified Eagle medium (DMEM) supplemented with $10 \%$ fetal bovine serum and $1 \%$ penicillin-streptomycin at $37^{\circ} \mathrm{C}$ with $5 \% \mathrm{CO}_{2}$, in accordance with a previously described protocol. $^{13}$

\section{Cohorts}

Formalin-fixed, paraffin-embedded, primary HCCs from 212 patients, who underwent initial surgical resection between March 2003 and August 2006, were randomly selected from the archives of the Department of Pathology of the First Affiliated Hospital, Sun Yat-Sen University (Guangzhou, China). We used these patients as a testing cohort. This cohort of patients with HCC included 174 (82\%) men and 38 (18\%) women, with a median age of 48 years, and their clinico-pathological characteristics are summarised in table 1. Average follow-up time was 28.79 months (median, 22.5 months; range, 1.0-81.0 months).

In parallel, we assessed another randomly collected, independent validation cohort of 163 patients diagnosed with liver disease between July 2005 and May 2008. These patients, whose diseases encompassed $126 \mathrm{HCCs}$ and 37 DNs, underwent initial surgical treatment at Sun Yat-Sen University Cancer Center (Guangzhou, China). The 126 patient with HCC included 95 (75.4\%) men and $31(24.6 \%)$ women, with a median age of 49.5 years (table 1 ). Average duration of follow-up was 23.69 months (median, 23.5 months; range, $1.0-53.0$ months). In this cohort, the 37 cases of DNs included 12 (32.4\%) women and 25 (67.6\%) men. The ages of the patients ranged from 19 to 72 years, and the mean age was 42.3 years. Patients with HCC were selected for both the testing and validation cohorts only if they had been given a distinctive pathological diagnosis, were undergoing primary and curative resection, and had not received preoperative anticancer treatment. Availability of patients' resection tissues and followup data were also criteria for selection.

We collected an additional cohort of 129 cases of 18-gauge needle biopsy specimens with hepatic nodules $(<3 \mathrm{~cm})$ from March 2007 to May 2010 in our institutes and from the Department of Pathology, Guangdong Provincial People's Hospital, (Guangzhou, China). To ensure that the pathological diagnosis of this cohort was accurate, only cases in which the patients subsequently underwent surgical resection were selected. The diagnoses of these biopsy tissues were confirmed as identical to those of the resultant surgically resected specimens. Of the 129 cases of liver biopsy, 72 were diagnosed as HCC, 25 as DN, 20 as focal nodular hyperplasia (FNH), six as hepatocellular adenoma (HA) and six as large regenerative nodule (LRN).

In this study, all diagnoses were formulated by expert pathologists (H-LR and Q-LW), according to the criteria for terminology established by the International Working Party. ${ }^{23}$ Tumour differentiation was based on the criteria of the World Health Organization Classification of Tumours (2000). Tumour stage was defined according to the 2002 American Joint Committee on Cancer/International Union Against Cancer tumour-node-metastasis (TNM) classification system. ${ }^{24}$ In addition, for EZH2 western blotting analysis, fresh tissue specimens from 10 patients with HCC who underwent surgical resection were collected in 2009 .

\section{Western blotting analysis}

Equal amounts of whole cell and tissue lysates were resolved by SDS-polyacrylamide gel electrophoresis (PAGE) and electrotransferred on a polyvinylidene difluoride (PVDF) membrane (Pall, Port Washington, New York, USA). The tissues were then incubated with primary mouse monoclonal antibodies against human EZH2 (1:1000 dilution; BD Transduction Laboratories, Franklin Lakes, New Jersey, USA). The immunoreactive signals were detected with enhanced chemiluminescence kit (Amersham Biosciences, Uppsala, Sweden). The procedures followed were conducted in accordance with the manufacturer's instructions 
Table 1 Association of enhancer of zeste homolog $2(\mathrm{EZH} 2)$ expression with patients' clinicopathological features in primary hepatocellular carcinomas

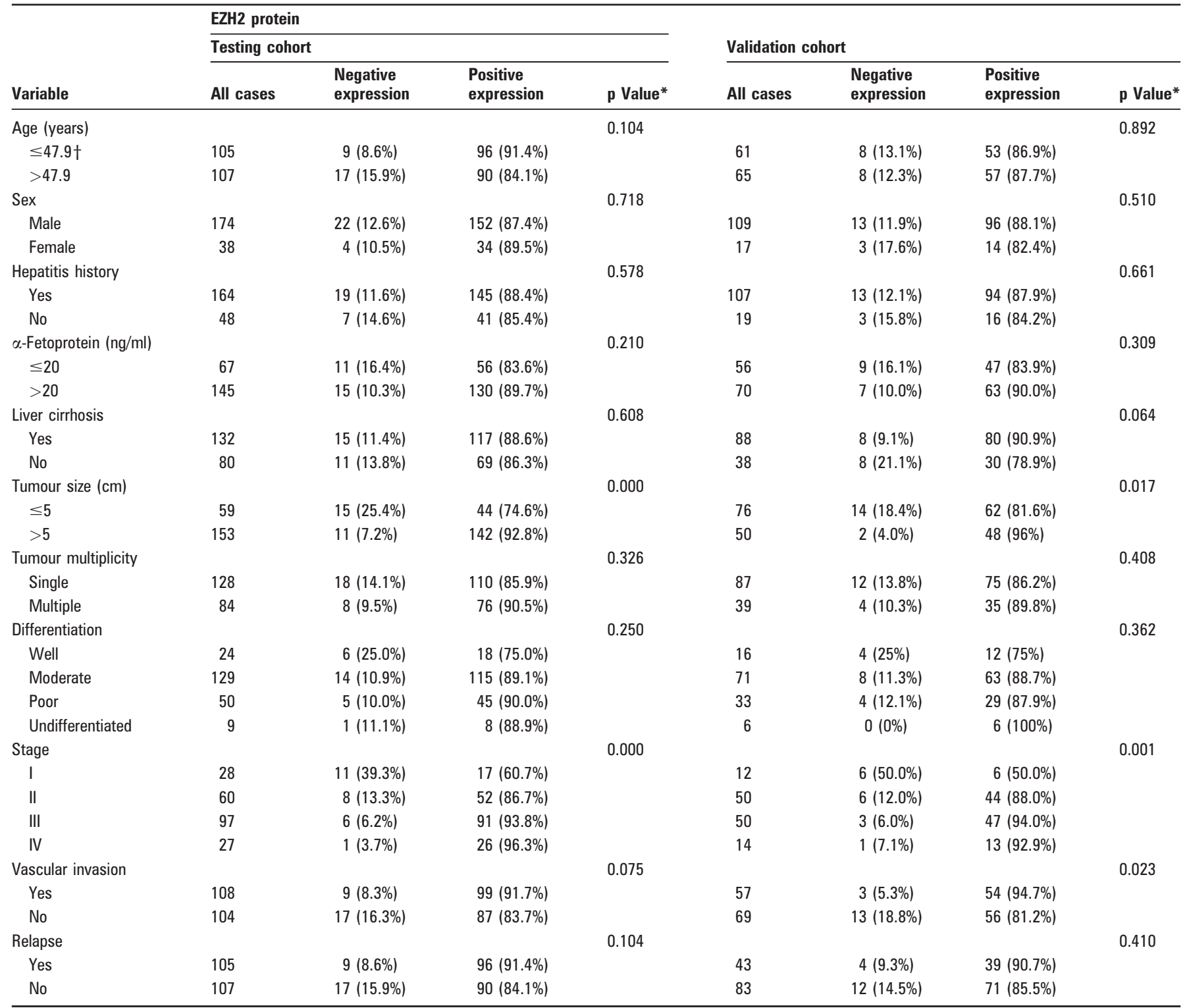

${ }^{*} \chi^{2}$ test.

†Mean age.

Tissue microarray and immunohistochemistry

Tissue microarrays (TMAs) were constructed in accordance with a previously described method. ${ }^{25}$ Triplicate $0.6 \mathrm{~mm}$ diameter cylinders (two identical cylinders taken from intra-tumoural tissue and one cylinder from peritumoural tissue) were punched from representative areas of an individual donor tissue block, and re-embedded into a recipient paraffin block in a defined position, using a tissue arraying instrument (Beecher Instruments, Silver Spring, Maryland, USA).

The TMA blocks were cut into 5 - $\mu \mathrm{m}$ sections and processed for IHC in accordance with a previously described protocol. ${ }^{26} \mathrm{TMA}$ slides were incubated respectively with anti-EZH2 (1:100 dilution; BD Transduction Laboratories, Franklin Lakes, New Jersey, USA), anti-HSP70 (1:200 dilution; Santa Cruz Biotechnology, Santa Cruz, California, USA) and anti-GPC3 (1:100 dilution; BioMosaics, Burlington, Vermont, USA), and stored overnight at $4^{\circ} \mathrm{C}$. Immunostaining was performed using the Envision System with diaminobenzidine (Dako, Glostrup, Denmark). A negative control was obtained by replacing the primary antibody with a normal murine or rabbit IgG. In the case of non-informative TMA samples (ie, samples with $<500$ tumour cells per case and lost samples), IHC staining was performed by using whole tissue slides.

\section{Evaluation by immunohistochemistry}

Immunoreactivity for EZH2, HSP70 and GPC3 proteins was scored using a semi-quantitative method by evaluating the number of positive tumour cells over the total number of tumour cells. Scores were assigned by using $5 \%$ increments $(0 \%$, $5 \%, 10 \% \ldots 100 \%$ ), as in our previous study. ${ }^{26}$ Expression for the markers was assessed by three independent pathologists (M-YC, H-LR and DX), who were blinded to the clinicopathological data. Their conclusions agreed in approximately $81 \%$ of the cases, indicating that this scoring method is highly reproducible. If two or three assessments were consistent with the results they scored, that value was selected. In cases where completely different results occurred, the three assessors discussed their assessments and agreed on the appropriate score to award. 


\section{Statistical analysis}

Statistical analyses were performed using the SPSS software program (SPSS Standard version 13.0). Receiver operating characteristic (ROC) curve analysis was applied to determine the cut-point for marker positivity by the 0,1-criterion, and sensitivity, specificity and the areas under the ROC curves (AUC) were calculated. To combine the three markers (EZH2, HSP70 and GPC3), we found the linear coefficient to maximise AUC for the combination. The associations between EZH2 expression and other variables were analysed by using the Spearman rank test. The statistical significance of the correlation between biomarker expression and disease-specific survival was estimated by the log-rank test. Multiple Cox proportional hazards regression was carried out to identify the independent factors which had a significant impact on patient survival. A difference was considered significant if the $p$ value from a two-tailed test was less than 0.05

\section{RESULTS}

Protein expression levels of EZH2 in liver cell lines and tissues by western blotting analysis

Western blotting analysis revealed an obviously higher level of EZH2 expression in all six HCC cell lines than in normal liver cell line Lo2, which was used for purposes of comparison (figure 1A, left). In liver tissues, upregulated expression of EZH2 was noticed in all cases of primary HCC tissues, while the level of expression of EZH2 was negligible in the adjacent non-neoplastic liver tissues (figure 1A, right).
Figure 1 The expression of enhancer of zeste homolog 2 (EZH2) in human hepatocellular cells and tissues. (A) The six hepatocellular carcinoma (HCC) cell lines, Huh7, HepG2, 7402, 7721, Нер3B and $\mathrm{Lm} 3$, exhibited higher levels of EZH2 expression, as determined by western blotting, than normal liver cell line Lo2. Upregulated expression of EZH2 was detected in all 10 cases of primary HCC tissues compared to adjacent non-neoplastic liver tissues. T, hepatocellular carcinoma tissue; N, non-neoplastic liver tissue. (B) Positive expression of EZH2 was examined by immunohistochemistry in HCC case No. 69. The adjacent non-neoplastic liver tissues (case 69) and a dysplastic nodule (DN) (case 9) negatively expressed EZH2 (upper panels, H\&E staining; lower panels,

immunohistochemical staining; $\times 100$ ). (C) Left: the box plot shows the mean staining intensity of EZH2 from HCC and non-neoplastic liver in the testing cohort $(\mathrm{p}<0.0001)$. Right: ROCs curves of EZH2 (area under curve $(A U C)=$ $0.990, p<0.0001$ ) in HCCs compared to adjacent non-neoplastic liver tissues in the testing cohort. (D) Left: the box plots demonstrate the range of $\mathrm{EZH} 2$ expression within each group (HCC, $n=126 ; \mathrm{DN}, n=37$; non-neoplastic liver tissues, $n=126)$ in the validation cohort ( $p<0.0001)$. Right: ROC curves comparing EZH2

(AUC $=0.935, p<0.0001$ ),

HSP70 (AUC $=0.936, p<0.0001)$, GPC3

( $A U C=0.890, p<0.0001)$ and the combination of the three markers ( $A \cup C=0.957, p<0.0001)$ in patients with HCC versus adjacent nonneoplastic liver tissues and DN. ROC, receiver operating characteristics.
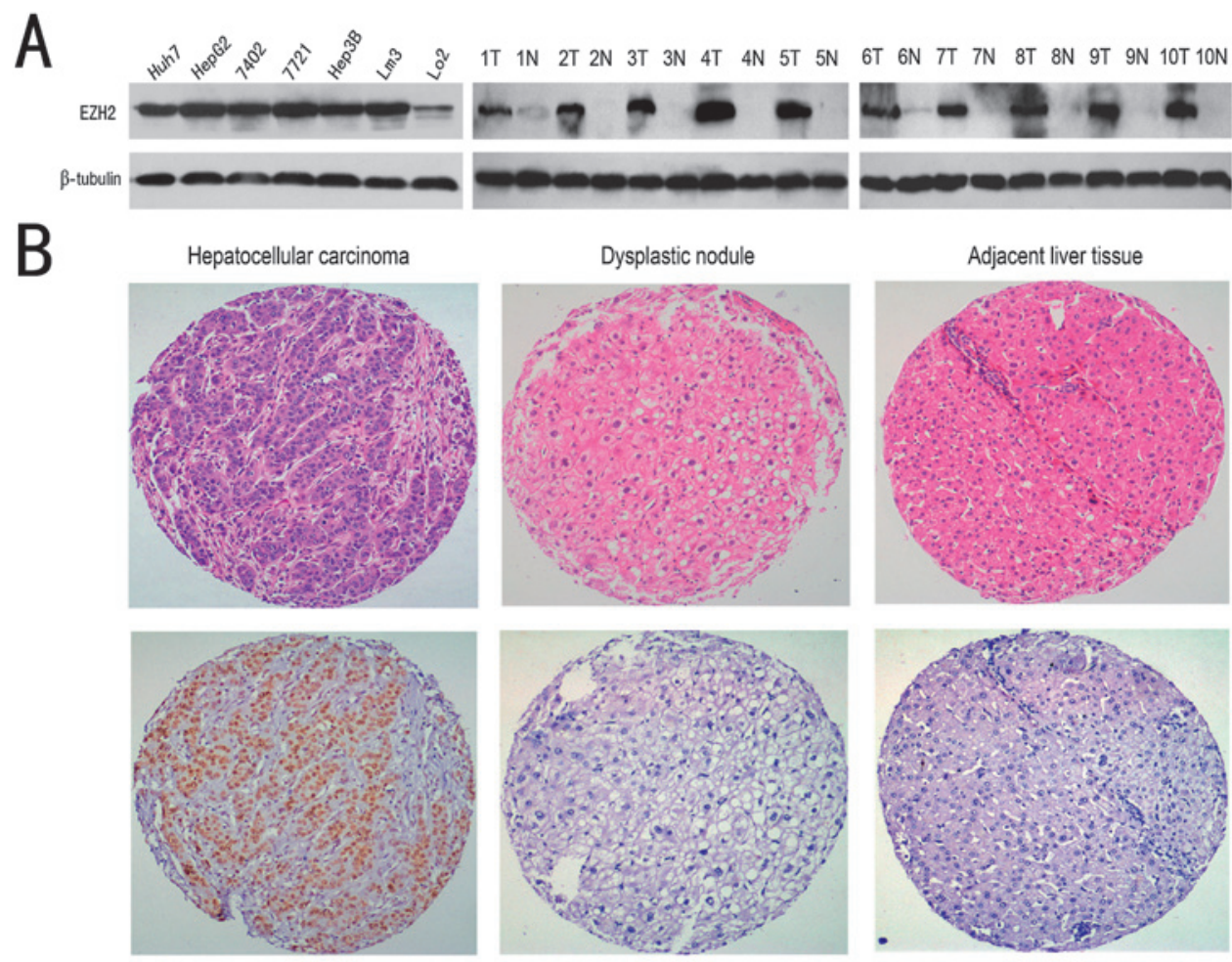

C
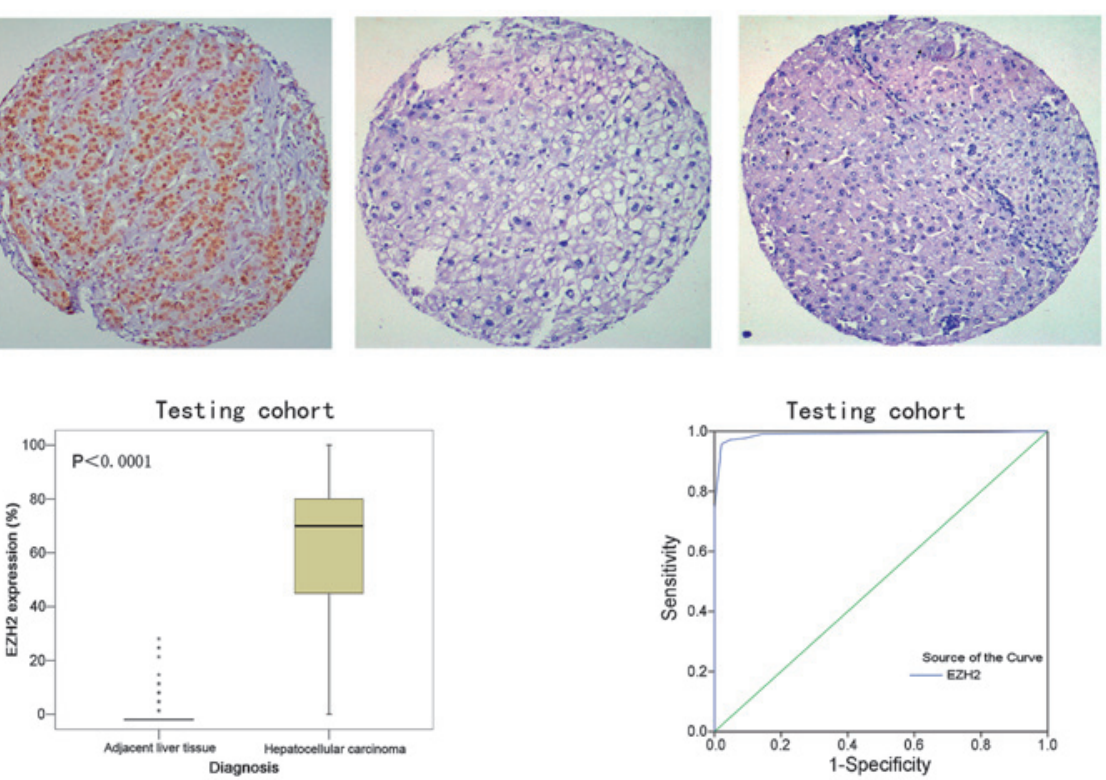

D
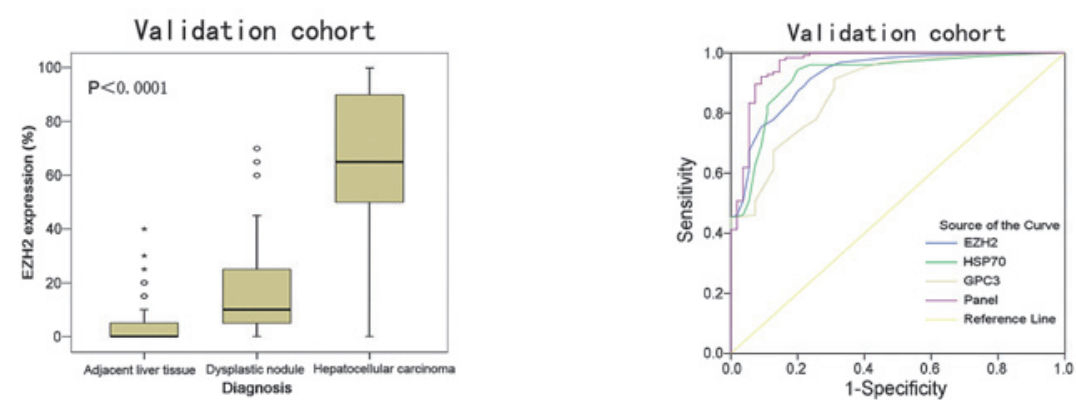
Identification of expression pattern of EZH2 in HCC tissue by IHC The degree of immunoreactivity of EZH2, which was observed primarily in the hepatocellular cell nuclei, ranged from $0 \%$ to $100 \%$ (figure 1B). Semi-quantification of primary HCC $(n=212)$ and adjacent non-neoplastic liver tissue $(n=212)$ in our testing cohort demonstrated a mean EZH2 staining intensity of $65.94 \%$ (SE, $1.652 \%$; $95 \%$ CI $62.69 \%$ to $69.20 \%$ ) and $2.41 \%$ (SE, $0.492 \%$; $95 \%$ CI $1.44 \%$ to $3.38 \%$ ), respectively (Wilcoxon exact test, $\mathrm{p}<0.0001$, figure $1 \mathrm{C}$, left). Overall, the sensitivity, specificity and AUC values of EZH2 expression levels for HCC detection versus non-neoplastic liver tissues were $95.8 \%, 97.8 \%$ and 0.990 , respectively $(p<0.0001$, figure $1 \mathrm{C}$, right). These values indicate that EZH2 is a potential diagnostic immunomarker for HCC.

\section{Validation of EZH2 as an IHC diagnostic marker for HCC}

To determine the reproducibility of these findings, the expression dynamics of EZH2 in HCC were next evaluated in our validation cohort, which included 126 HCCs and 37 DNs, by IHC. In this cohort, the mean staining intensity of EZH2 in HCCs was $63.73 \%$ (SE, 2.259\%; 95\% CI $59.26 \%$ to $68.20 \%$ ), which was significantly higher than those in the adjacent nonneoplastic liver tissues (mean, 4.01\%; SE, 0.647\%; 95\% CI $2.73 \%$ to $5.29 \%$ ) and in DNs (mean, $17.16 \%$; SE, $3.139 \%$; $95 \%$ CI $10.80 \%$ to $23.53 \%$; Wilcoxon exact test, $p<0.0001$, figure $1 \mathrm{D}$, left). Further analysis also showed a significant difference in the expression percentages of EZH2 between the WD HCCs (mean, $60.81 \%$ ) and the DNs (mean, 17.16\%, Wilcoxon exact test, $\mathrm{p}<0.0001)$. Overall, the sensitivity, specificity and AUC for the diagnosis of HCC versus DN/non-neoplastic liver tissue using the expression levels of EZH2 above were $83.3 \%, 90.9 \%$ and 0.935 , respectively $(p<0.0001$, figure $1 \mathrm{D}$, right).

Since HSP70 and GPC3 have recently been suggested as promising biomarkers for distinguishing between malignant and non-malignant hepatocellular lesions, ${ }^{17} 20$ we also compared the diagnostic performance of EZH2 for HCC with that of HSP70 and GPC3. We further investigated whether a combination of these three potential markers improved their ability to diagnose HCC. The results demonstrated that HSP70 and GPC3 immunoreactivity were primarily examined in the nucleocytoplasm and cytoplasm, respectively (figure 2A). The sensitivity, specificity and AUC values for HCC diagnosis were $82.8 \%, 92.7 \%$ and 0.936 when HSP70 was used, and $77.0 \%, 90.4 \%$ and 0.890 when GPC3 was used $(p<0.0001$, figure $1 \mathrm{D}$, right $)$. Strikingly, the sensitivity, specificity and AUC values increased to $89.7 \%, 94.5 \%$ and 0.957, when EZH2 was combined with HSP70 and GPC3 $(p<0.0001$, figure 1D, right). Furthermore, for the detection of WD HCCs, the sensitivity and specificity of individual markers or a combination were $68.9 \%$ and $91.5 \%$ for $\mathrm{EZH} 2,62.5 \%$ and $98.5 \%$ for HSP70, $50.0 \%$ and $92.1 \%$ for GPC3, and $75.0 \%$ and $100 \%$ for the three-marker panel (ie, a combination of EZH2, HSP70 and GPC3). A comparison for ROC curves showed that the AUC for the three-marker combination (EZH2, HSP70 and GPC3) was significantly larger than that for GPC3 alone $(p=0.010)$, while there were no significant differences of AUC between the threemarker combination and EZH2 or HSP70 alone and between each of the three markers $(p>0.05)$. These findings indicate (a) that EZH2 alone is more sensitive for the diagnosis of WD HCC than either HPS70 or GPC3, and (b) that the combination of the three markers could greatly improve the diagnostic accuracy of HCC, particularly for WD HCC detection (figure 2B).

\section{Potential diagnostic utility of EZH2, HSP70 and GPC3 for HCC detection in liver needle biopsies}

In clinical settings, a needle biopsy is normally recommended to identify the features of malignancy when small hepatic nodules are detected. Additional biomarkers for the discrimination of WD HCC and DN are needed. ${ }^{27}$ To evaluate the diagnostic values of EZH2 and the three-marker combination (EZH2,

A

$H \& E$

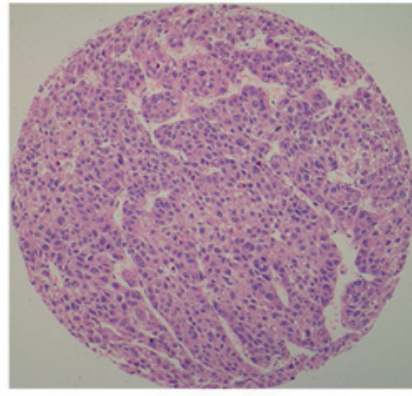

$H \& E$

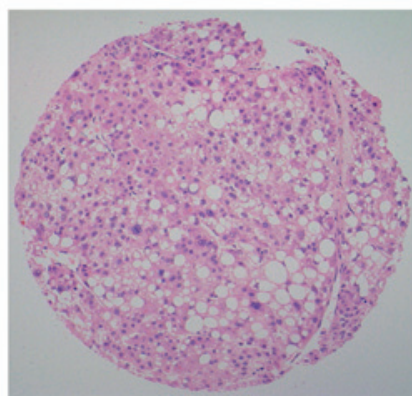

$\mathrm{EZH2}$

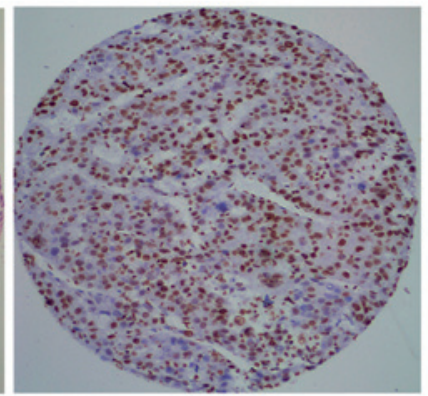

EZH2

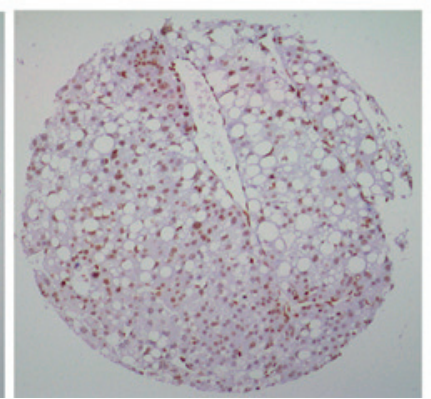

HSP7O

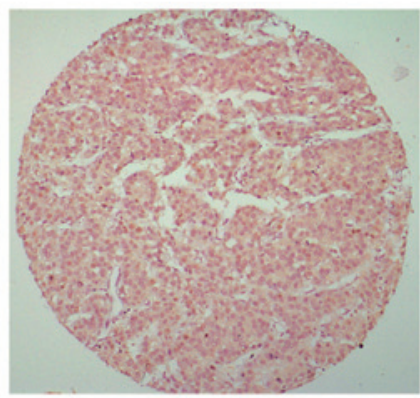

HSP7O

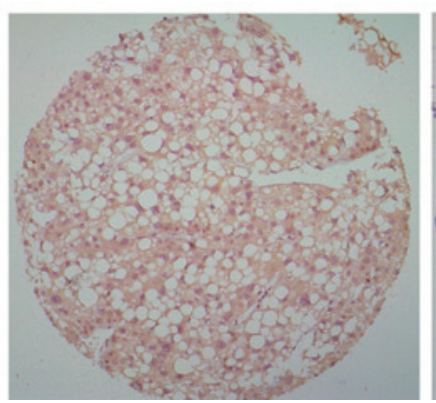

GPC3

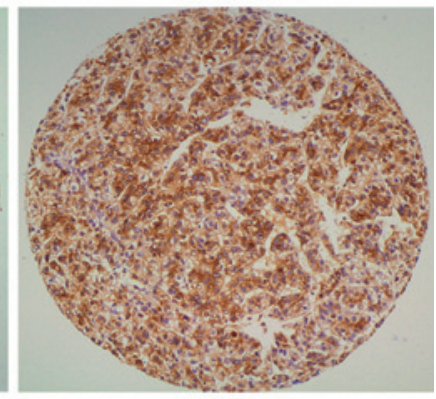

GPC3

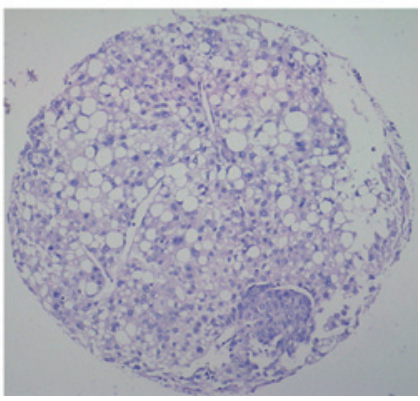

Figure 2 The expression dynamics of enhancer of zeste homolog 2 (EZH2), heat shock protein 70 (HSP70) and glypican 3 (GPC3) examined by immunohistochemistry in hepatocellular carcinoma (HCC) resection tissues. (A) A moderately differentiated HCC case positively expresses EZH2, HSP70 and GPC3. (B) A well-differentiated HCC case positively expresses EZH2 and HSP70, but negatively expresses GPC3. 
A

H\&E

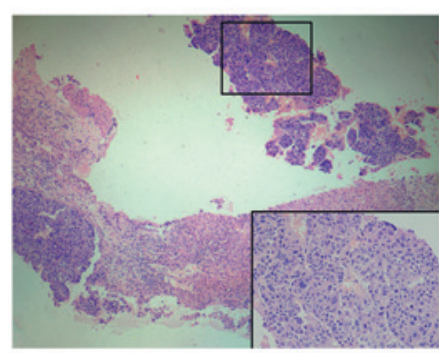

EZH2

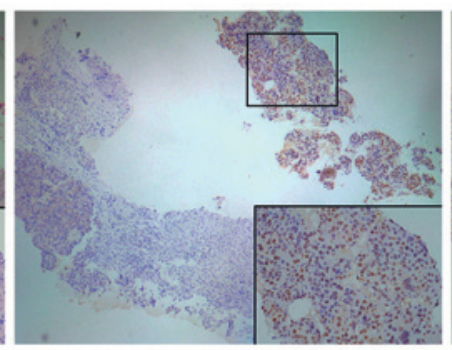

HSP7O

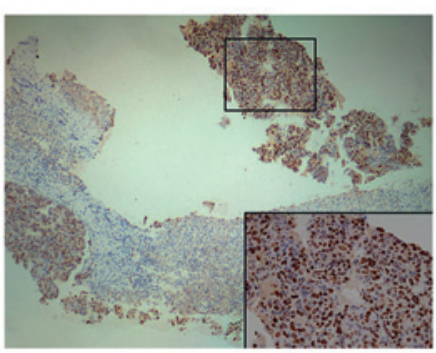

GPC3

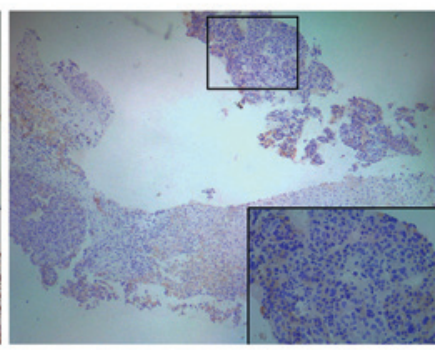

B

H\&E

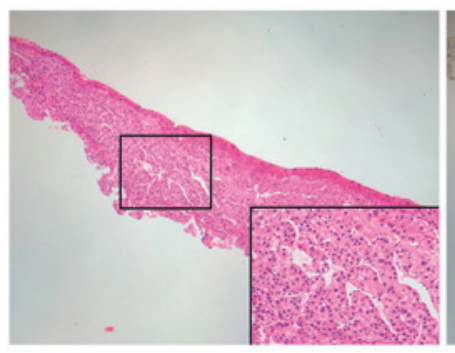

EZH2

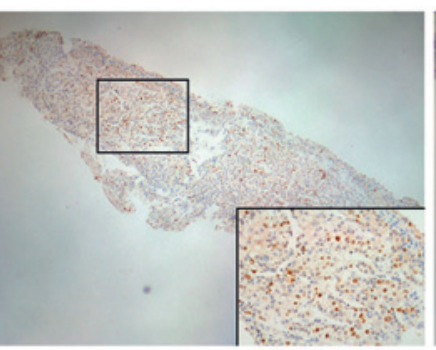

HSP70

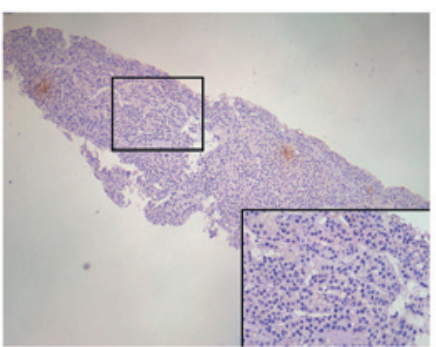

GPC3

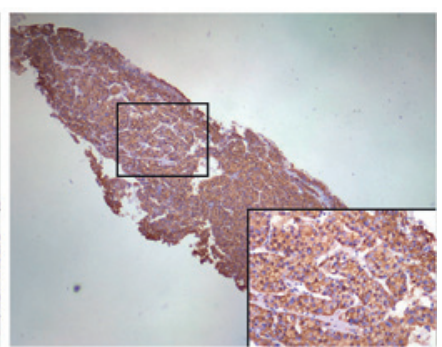

C

H\&E

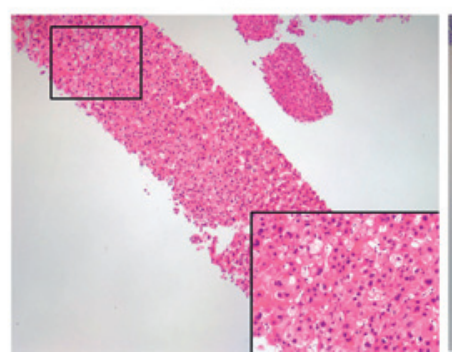

EZH2

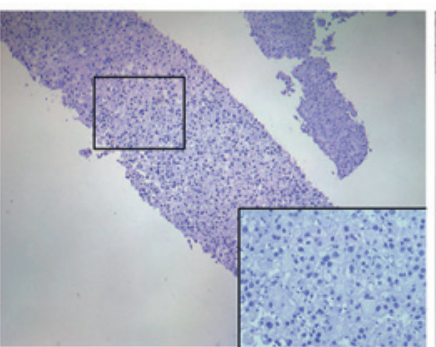

HSP7O

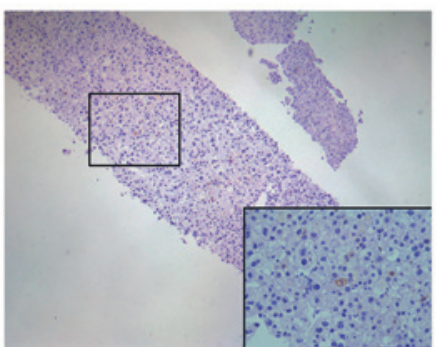

GPC3

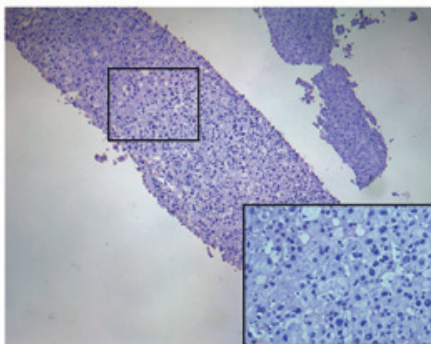

Figure 3 The expression patterns enhancer of zeste homolog 2 (EZH2), heat shock protein 70 (HSP70) and glypican 3 (GPC3) examined by immunohistochemistry in liver needle biopsy tissues. (A) A poorly differentiated hepatocellular carcinoma (HCC) biopsy tissue positively expresses EZH2, HSP70 and GPC3, while the adjacent non-neoplastic liver tissues show negative staining of the three markers. (B) A moderately differentiated HCC biopsy case shows positive immunostaining of EZH2 and GPC3, but only weak immunostaining by HSP70. (C) A dysplastic nodule biopsy case negatively expresses EZH2, HSP70 and GPC3. Representative sites in liver needle biopsy tissue at low $(\times 100)$ and high (inset, $\times 400)$ magnification are shown.

HSP70 and GPC3) in liver needle biopsy samples, we further performed IHC examinations of EZH2, HSP70 and GPC3 in an additional cohort of 129 biopsy specimens with hepatic nodules. To identify a single, optimal cut-point for positivity, ROC curve analysis was applied to the cohort of surgical resection tissues to determine the cut-off score for positive expressions of the three markers concerned. The score closest to the point with both maximum sensitivity and specificity, that is, the point $(0.0,1.0)$ on the curve, was selected as the cut-off score, leading to the greatest number of patients correctly classified as HCC or non-

Table 2 Immunohistochemical analysis of the different lesions in liver needle biopsies

\begin{tabular}{|c|c|c|c|c|c|c|c|}
\hline & $\begin{array}{l}\text { LRN } \\
n=6\end{array}$ & $\begin{array}{l}\text { HA } \\
n=6\end{array}$ & $\begin{array}{l}\text { FNH } \\
n=20\end{array}$ & $\begin{array}{l}D N \\
n=25\end{array}$ & $\begin{array}{l}\text { WD HCC } \\
n=18\end{array}$ & $\begin{array}{l}\text { MD HCC } \\
n=37\end{array}$ & $\begin{array}{l}\text { PD HCC } \\
n=17\end{array}$ \\
\hline All three positive & 0 & 0 & 0 & 0 & 4 & 16 & 6 \\
\hline At least two positive & 0 & 0 & 0 & 0 & 11 & 32 & 15 \\
\hline At least one positive & 0 & 0 & 2 & 7 & 16 & 37 & 17 \\
\hline EZH2+/HSP70+ & 0 & 0 & 0 & 0 & 7 & 22 & 8 \\
\hline $\mathrm{EZH} 2+/ \mathrm{GPC} 3+$ & 0 & 0 & 0 & 0 & 6 & 21 & 10 \\
\hline HSP70+/GPC3+ & 0 & 0 & 0 & 0 & 6 & 21 & 9 \\
\hline $\mathrm{EZH} 2+$ & 0 & 0 & 1 & 3 & 12 & 31 & 13 \\
\hline HSP70+ & 0 & 0 & 1 & 2 & 10 & 28 & 11 \\
\hline $\mathrm{GPC} 3+$ & 0 & 0 & 0 & 2 & 9 & 26 & 14 \\
\hline
\end{tabular}

DN, dysplastic nodule; FNH, focal nodular hyperplasia; HA, hepatocellular adenoma; LRN, large regenerative nodule; MD HCC, moderately differentiated hepatocellular carcinoma; PD HCC, poorly differentiated hepatocellular carcinoma; WD HCC, welldifferentiated hepatocellular carcinoma. 
Table 3 Degree of diagnostic accuracy in HCC and non-malignant (NM) nodules (whole series)

\begin{tabular}{|c|c|c|c|c|c|c|c|c|}
\hline & $\begin{array}{l}\text { HCC } \\
(n=72)\end{array}$ & $\begin{array}{l}\text { NM } \\
(n=57)\end{array}$ & Sensitivity & Specificity & PPV & NPV & Accuracy & $\begin{array}{l}\text { Youden } \\
\text { index }\end{array}$ \\
\hline All three positive & 26 & 0 & $36.1 \%$ & $100 \%$ & $100 \%$ & $55.3 \%$ & $64.3 \%$ & 0.36 \\
\hline At least two positive & 58 & 0 & $80.6 \%$ & $100 \%$ & $100 \%$ & $80.3 \%$ & $89.2 \%$ & 0.81 \\
\hline At least one positive & 70 & 9 & $97.2 \%$ & $84.2 \%$ & $88.6 \%$ & $96.0 \%$ & $91.5 \%$ & 0.81 \\
\hline $\mathrm{EZH} 2+/ \mathrm{HSP} 70+$ & 37 & 0 & $51.4 \%$ & $100 \%$ & $100 \%$ & $62.0 \%$ & $72.9 \%$ & 0.51 \\
\hline $\mathrm{EZH} 2+/ \mathrm{GPC} 3+$ & 37 & 0 & $51.4 \%$ & $100 \%$ & $100 \%$ & $62.0 \%$ & $72.9 \%$ & 0.51 \\
\hline HSP70+/GPC3+ & 36 & 0 & $50.0 \%$ & $100 \%$ & $100 \%$ & $61.3 \%$ & $72.1 \%$ & 0.50 \\
\hline $\mathrm{EZH} 2+$ & 56 & 4 & $77.8 \%$ & $93.0 \%$ & $93.3 \%$ & $76.8 \%$ & $84.5 \%$ & 0.71 \\
\hline HSP70+ & 49 & 3 & $68.1 \%$ & $94.7 \%$ & $94.2 \%$ & $70.1 \%$ & $79.8 \%$ & 0.63 \\
\hline GPC3+ & 49 & 2 & $68.1 \%$ & $96.5 \%$ & $94.2 \%$ & $70.5 \%$ & $80.6 \%$ & 0.65 \\
\hline
\end{tabular}

HCC. Thus, tumours designated positive for EZH2, HSP70 and GPC3 were those with scores above the value of 30\%, 10\% and $10 \%$, respectively. By using these criteria, in our biopsy tissues, all 12 cases of LRN/HA were negatively stained by each of the three markers, whereas the number of immuno-positive cases for which there was at least one marker increased from 2/20 (10.0\%) in the case of FNHs to $7 / 25$ (28.0\%) for DNs and to 16/18 (88.9\%) for WD HCCs; and to $54 / 54$ (100.0\% in the case of moderately differentiated $(\mathrm{MD})+$ poorly differentiated $(\mathrm{PD}) \mathrm{HCC}$ (figure $3 \mathrm{~A}, \mathrm{~B}$, table 2). Immuno-positive cases for which there were at least two markers (regardless of their identity) were observed in $0 / 6 \mathrm{LRNs}$, in 0/6 HAs, in 0/20 FNHs, in 0/25 DNs (figure 3C), in $11 / 18$ (61.1\%) WD HCCs, in 32/37 (86.5\%) MD HCCs, and in 15/ $17(88.2 \%)$ PD HCCs (table 2$)$. Further statistical analysis showed that when at least one positive marker was considered, the sensitivity, specificity, positive predictive value (PPV), negative predictive value (NPV), accuracy and Youden index for differentiating HCCs from non-malignant nodules were 97.2\%, 84.2\%, $88.6 \%, 96.0 \%, 91.5 \%$ and 0.81 , respectively. When at least two positive markers were used, the sensitivity, specificity, PPV, NPV, accuracy and Youden index were $80.6 \%, 100 \%, 100 \%, 80.3 \%$, $89.2 \%$ and $0.81 \%$, respectively (table 3 ).

\section{Association between EZH2 expression and clinico-pathological features and survival of patients with HCC}

Using the ROC curve of EZH2 described before, the positive expression of EZH2 was examined in 186/212 (87.7\%) of HCCs in the testing cohort and in 110/126 (87.3\%) of HCCs in the validation cohort, respectively. Further correlation analysis in both cohorts showed that the positive expression of EZH2 in HCCs was significantly associated with a more aggressive phenotype of the tumour $(p<0.05$, table 1). Kaplan-Meier analysis established that, in the testing cohort, the median disease-specific survival time for patients with HCC who positively expressed EZH2 was 34.8 months, compared to 66.5 months for patients with HCC who negatively expressed $\mathrm{EZH} 2(\mathrm{p}=0.001$, log-rank test, figure 4A, table 4$)$. In the validation cohort, patients with HCC who positively expressed EZH2 also exhibited a shorter survival time (median survival time, 33.5 months) than patients with HCC who negatively expressed $\mathrm{EZH} 2$ (median, 49.2 months; $\mathrm{p}=0.009$, log-rank test, figure $4 \mathrm{~B}$, table 4). Further multivariate Cox regression analysis indicated that EZH2 expression is an independent prognostic factor for poor survival of HCC patients in both cohorts (table 5).

\section{DISSCUSSION}

The polycomb group protein EZH2 has recently been suggested to play a crucial role in the tumourigenesis of several types of human cancer, including HCC. ${ }^{10-12}$ We previously found that the knocking down of EZH2 by shRNA can significantly inhibit the growth of HCC cells in vitro and their tumourigenicity in vivo. ${ }^{13}$ Several reports noted that EZH2 was over-expressed in most of the HCC resection tissues by IHC, whereas it was negatively expressed in nearly all the corresponding non-tumour tissues and DNs. ${ }^{14} 16$ These observations indicate that it may be possible to use the expression levels of EZH2 as a diagnostic tool to distinguish HCC tissues from non-malignant liver tissues. To date, however, studies of the use of EZH2 in HCC cases have defined EZH2 protein expression using a predetermined and arbitrary cut-off score. Furthermore, no investigation and validation studies are available.

In the present study, our initial western blotting established that EZH2 was expressed at a higher level in a panel of HCC cell lines and tissues than in a hepatic cell line and adjacent liver tissues. We next used IHC staining to examine the expression dynamics of EZH2 in two large independent (testing and validation) cohorts of HCCs and non-malignant liver tissues. According to our results, the mean staining intensity of EZH2 in HCCs in both cohorts was significantly greater than in non-
Figure 4 Kaplan-Meier survival analysis according to enhancer of zeste homolog $2(\mathrm{EZH} 2)$ protein expression in 338 patients with hepatocellular carcinoma (log-rank test). (A) Probability of survival of patients in testing cohort: negative expression of EZH2, $n=26$; positive expression of $\mathrm{EZH} 2, n=186(\mathrm{p}=0.001)$. (B) Probability of survival of patients in validation cohort: negative expression of EZH2, $n=16$; positive expression of $\mathrm{EZH} 2, n=110(\mathrm{p}=0.009)$.
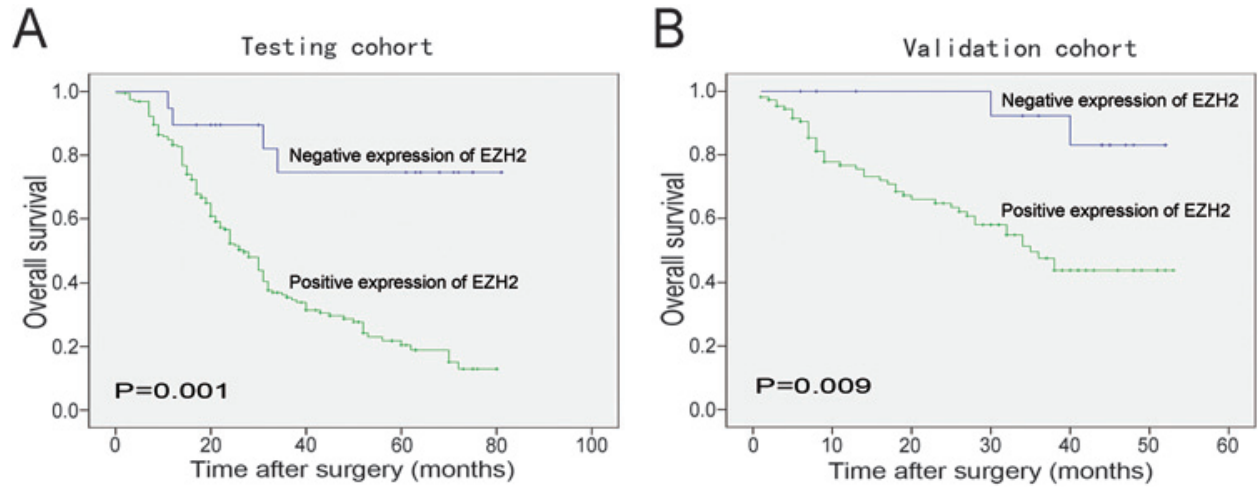
Table 4 Univariate analysis of enhancer of zeste homolog 2 (EZH2) expression and various clinicopathological parameters in 338 patients with primary hepatocellular carcinoma

\begin{tabular}{|c|c|c|c|c|c|c|}
\hline \multirow[b]{2}{*}{ Variable } & \multicolumn{3}{|c|}{ Testing cohort } & \multicolumn{3}{|c|}{ Validation cohort } \\
\hline & All cases & HR (95\% CI) & p value* & All cases & HR $(95 \% \mathrm{CI})$ & p Value* \\
\hline Age (years) & & & 0.627 & & & 0.721 \\
\hline$\leq 47.9 \dagger$ & 105 & 1.0 & & 61 & 1.0 & \\
\hline$>47.9$ & 107 & $1.090(0.770$ to 1.542$)$ & & 65 & $1.109(0.628$ to 1.959$)$ & \\
\hline Sex & & & 0.045 & & & 0.558 \\
\hline Male & 174 & 1.650 (1.012 to 2.688$)$ & & 109 & $1.293(0.548$ to 3.052$)$ & \\
\hline Female & 38 & 1.0 & & 17 & 1.0 & \\
\hline Hepatitis history & & & 0.810 & & & 0.991 \\
\hline Yes & 164 & $1.053(0.692$ to 1.602$)$ & & 107 & $1.004(0.450$ to 2.240$)$ & \\
\hline No & 48 & 1.0 & & 19 & 1.0 & \\
\hline AFP (ng/ml) & & & 0.000 & & & 0.000 \\
\hline$\leq 20$ & 67 & 1.0 & & 56 & 1.0 & \\
\hline$>20$ & 145 & $2.262(1.490$ to 3.434$)$ & & 70 & 5.305 (2.826 to 9.958$)$ & \\
\hline Liver cirrhosis & & & 0.891 & & & 0.983 \\
\hline Yes & 132 & 0.975 (0.682 to 1.395$)$ & & 88 & 1.0 & \\
\hline No & 80 & 1.0 & & 38 & $0.993(0.539$ to 1.831$)$ & \\
\hline Tumour size $(\mathrm{cm})$ & & & 0.000 & & & 0.000 \\
\hline$\leq 5$ & 59 & 1.0 & & 76 & 1.0 & \\
\hline$>5$ & 153 & 6.295 (3.688 to 10.747$)$ & & 50 & $2.839(1.586$ to 5.082$)$ & \\
\hline Tumour multiplicity & & & 0.000 & & & 0.000 \\
\hline Single & 128 & 1.0 & & 87 & 1.0 & \\
\hline Multiple & 84 & $3.480(2.429$ to 4.987$)$ & & 39 & 3.672 (2.065 to 6.529$)$ & \\
\hline Differentiation & & & 0.021 & & & 0.102 \\
\hline Well-moderate & 153 & 1.0 & & 87 & 1.0 & \\
\hline Poor-undifferentiated & 59 & 1.534 (1.068 to 2.203$)$ & & 39 & $1.630(0.908$ to 2.927$)$ & \\
\hline Stage & & & 0.000 & & & 0.000 \\
\hline I-II & 88 & 1.0 & & 62 & 1.0 & \\
\hline III-IV & 124 & $5.393(3.525$ to 8.252$)$ & & 64 & $5.400(2.526$ to 11.542$)$ & \\
\hline Vascular invasion & & & 0.000 & & & 0.000 \\
\hline Yes & 108 & $4.923(3.310$ to 7.322$)$ & & 57 & $5.362(2.724$ to 10.553$)$ & \\
\hline No & 104 & 1.0 & & 69 & 1.0 & \\
\hline Relapse & & & 0.000 & & & 0.000 \\
\hline Yes & 105 & 1.907 (1.330 to 2.734$)$ & & 43 & $2.860(1.600$ to 5.112$)$ & \\
\hline No & 107 & 1.0 & & 83 & 1.0 & \\
\hline $\mathrm{EZH} 2$ & & & 0.001 & & & 0.009 \\
\hline Negative expression & 26 & 1.0 & & 16 & 1.0 & \\
\hline Positive expression & 186 & 3.506 (1.703 to 7.219$)$ & & 110 & 5.451 (1.318 to 22.543$)$ & \\
\hline
\end{tabular}

malignant liver tissues. The expression levels of EZH2 were able to distinguish HCCs from non-malignant liver tissues with very high sensitivity, specificity and AUC. Furthermore, the staining intensity of EZH2 in WD HCCs was significantly greater than that in DNs. These observations strongly suggest that the evaluation of EZH2 expression by IHC can enable us not only to discriminate HCCs and non-neoplastic liver tissues, but also to distinguish WD HCCs from DNs with a high degree of accuracy.

In clinical practice, an increasing number of small hepatocellular nodules $(<3 \mathrm{~cm})$ are detected by imaging during the followup of patients with liver cirrhosis, but the sensitivity of this imaging for the detection of small HCCs is only around $33 \%$. $^{28} 29$ Recently, liver needle biopsies have been recommended as a check on diagnoses of small hepatic nodules which were not satisfactorily addressed by imaging. However, since histological diagnosis by needle liver biopsy is based solely on the analysis of tiny fragments of the tissue, it is very difficult to distinguish between early WD HCCs and certain benign hepatocellular diseases such as DNs. This often results in diagnostic delays. ${ }^{30-33}$ A number of biomarkers have been suggested that can potentially distinguish HCCs from DNs in cirrhosis cases. The diagnostic yield of a panel of putative HCC markers, such as
HSP70, GPC3 and glutamine synthetase has been examined, and they have been proposed as useful immunomarkers for HCC. ${ }^{21} 22$ In this study, the diagnostic performance of HSP70 or GPC3 and their combination with EZH2 was first analysed in our surgical resection liver specimens. As expected, both HSP70 and GPC3 alone showed a high sensitivity, specificity and AUC for HCC detection. Interestingly, the sensitivity, specificity and AUC for HCC diagnosis increased when EZH2 was used in combination with HSP70 and GPC3. In addition, for the diagnosis of WD HCCs, the sensitivity and specificity of individual markers or a combination were $68.9 \%$ and $91.5 \%$ for EZH2, $62.5 \%$ and $98.5 \%$ for HSP70, $50.0 \%$ and $92.1 \%$ for GPC3, and $75.0 \%$ and $100 \%$ for the 3 -marker panel.

We wondered whether the expression status of EZH2 would be retained in liver needle biopsies, thereby also serving as a useful diagnostic immunomarker to distinguish between HCCs and benign hepatocellular tissues. We therefore re-evaluated the diagnostic values of EZH2 and the three-marker combination (EZH2, HSP70 and GPC3) in an additional cohort of liver needle biopsy samples. By using the cut-off score established in ROC curve analysis before, we found that in liver biopsies, LRNs and HAs were all stained negatively by each of the three markers, whereas 
Table 5 Cox multivariate analyses of prognostic factors on overall survival

\begin{tabular}{|c|c|c|}
\hline Characteristic & HR $(95 \% \mathrm{CI})$ & p Value \\
\hline \multicolumn{3}{|l|}{ The First Affiliated Hospital cohort } \\
\hline Sex (female vs male) & $1.303(0.793$ to 2.140$)$ & 0.297 \\
\hline$\alpha$-Fetoprotein, $\mathrm{ng} / \mathrm{ml}(\leq 20$ vs $>20$ ) & $1.398(0.912$ to 2.141$)$ & 0.124 \\
\hline Tumour size, $\mathrm{cm}(\leq 5$ vs $>5$ ) & 0.768 (0.437 to 1.348$)$ & 0.358 \\
\hline Tumour multiplicity (single vs multiple) & 1.711 (1.123 to 2.605$)$ & 0.012 \\
\hline $\begin{array}{l}\text { Differentiation (well-moderate vs } \\
\text { poor-undifferentiated) }\end{array}$ & 0.964 (0.518 to 1.792$)$ & 0.907 \\
\hline Stage $(I-I I$ vs III-V) & $1.818(1.313$ to 2.516$)$ & 0.000 \\
\hline Vascular invasion (no vs yes) & 2.609 (1.716 to 3.966$)$ & 0.000 \\
\hline Relapse (no vs yes) & $1.159(0.806$ to 1.667$)$ & 0.426 \\
\hline EZH2 (positive vs negative) & 2.305 (1.178 to 4.530$)$ & 0.032 \\
\hline \multicolumn{3}{|l|}{ The Cancer Center cohort } \\
\hline$\alpha$-Fetoprotein, $\mathrm{ng} / \mathrm{ml}(\leq 20$ vs $>20$ ) & $2.083(1.076$ to 4.033$)$ & 0.029 \\
\hline Tumour size, $\mathrm{cm}(\leq 5$ vs $>5$ ) & $1.627(0.836$ to 3.165$)$ & 0.152 \\
\hline Tumour multiplicity (single vs multiple) & $1.628(0.843$ to 3.144$)$ & 0.147 \\
\hline Stage (I-II vs III-IV) & $1.616(0.862$ to 3.031$)$ & 0.134 \\
\hline Vascular invasion (no vs yes) & $5.228(1.486$ to 18.390$)$ & 0.000 \\
\hline Relapse (no vs yes) & $1.163(0.606$ to 2.232$)$ & 0.650 \\
\hline EZH2 (positive vs negative) & $4.522(1.020$ to 20.054$)$ & 0.047 \\
\hline
\end{tabular}

immuno-positive cases for at least one marker increased from $\mathrm{FNH}$ $(2 / 20)$ and DN (7/25) to WD HCC (16/18), and to MD+PD HCC $(54 / 54)$. Using the three-marker panel, immuno-positive cases (ie, cases where any two markers showed positive) were observed in none of the benign liver lesions, but were frequently examined in $11 / 18$ WD HCCs, in 32/37 MD HCCs, and in 15/17 PD HCCs. Further statistical analysis determined that sensitivity was $97.2 \%$ when at least one marker was used, but only $80.6 \%$ when at least two markers were used. Specificity increased from $84.2 \%$ when at least one marker was used, to $100 \%$ when at least two markers were used; while the PPV increased from $88.6 \%$ to $100 \%$. The accuracy and Youden index remained nearly or exactly identical. These findings were intriguing, since PPV is highly useful in both marker situations as well as for NPV. Collectively, our findings not only confirm the value of EZH2 for the detection of HCC, but also demonstrate that the use of a three-marker panel (EZH2, HSP70 and GPC3) can improve the rate of detection of HCCs in liver biopsy tissues.

To sum up, in this study we describe the protein expression patterns of EZH2 in several cohorts of human HCCs and nonmalignant liver tissues, and demonstrate the potential utility of EZH2 as an immunomarker of HCCs during the diagnosis of liver lesions. We show, for the first time, that a three-marker panel (ie, EZH2, HSP70 and GPC3) is better able to identify HCCs in liver biopsy tissues than EZH2 alone. Our results also provide evidence that the positive expression of EZH2 in HCCs may be important for the detection of an aggressive phenotype or a phenotype with poor prognosis. We believe that the use of EZH2 protein, as examined by IHC, as a diagnostic biomarker of HCCs could improve the prospects of the early detection of HCCs in liver needle biopsies; and that an improved rate of detection would have important prognostic implications for patients with HCC. It is necessary to point out that our current study is of the retrospective nature. The main limitations of this study are that (a) patients with lesions confidently classified as HCC by biopsy and explant analysis were included; and (b) a high proportion of large lesions $(>5 \mathrm{~cm}$ ) was included in our surgically resected HCC cohorts to establish the diagnostic criteria of biomarkers, in which the diagnosis by haematoxylin\&eosin is usually not difficult. Clearly, further prospective studies designed to include HCC lesions smaller than $2 \mathrm{~cm}$ are needed to validate the conclusions of this study.
Acknowledgements We are grateful to Dr David Wilmshurst, Academic Editor of the Chinese University of Hong Kong, for commenting on a draft of this paper.

Funding This study was supported by grants made under China's 973 National Basic Research Programme (Nos. 2010CB529401 and 2010CB912803) and 863 High Technology Development Programme (No. 2007AA021901), and by a grant from the Guangzhou Science and Technology Bureau Foundation (No. 2005Z1-E0131).

\section{Competing interests None.}

Ethics approval This study was conducted with the approval of The Institute Research Medical Ethics Committee of Sun Yat-Sen University Cancer Center.

Contributors MYC, ZTT and FZ contributed equally to this study. Each author who contributed to this work has been listed as an author on the paper.

Provenance and peer review Not commissioned; externally peer reviewed.

\section{REFERENCES}

1. Ince $\mathbf{N}$, Wands JR. The increasing incidence of hepatocellular carcinoma. $N$ Engl $J$ Med 1999; 340:798-9.

2. El-Serag HB, Mason AC. Rising incidence of hepatocellular carcinoma in the United States. N Engl J Med 1999;340:745-50.

3. Hsu YC, Fu HH, Jeng YM, et al. Proline-directed protein kinase FA is a powerful and independent prognostic predictor for progression and patient survival of hepatocellular carcinoma. J Clin Oncol 2006;24:3780-8.

4. Bruix J, Sherman M. Management of hepatocellular carcinoma. Hepatology 2005; $42: 1208-36$.

5. Llovet JM, Burroughs A, Bruix J. Hepatocellular carcinoma. Lancet 2003:362:1907-17

6. Nomura F, Ishijima M, Kuwa K, et al. Serum des-gamma-carboxy prothrombin levels determined by a new generation of sensitive immunoassays in patients with smallsized hepatocellular carcinoma. Am J Gastroenterol 1999;94:650-4.

7. Chen ZMM, Crone KG, Watson MA, et al. Identification of a unique gene expression signature that differentiates hepatocellular adenoma from well-differentiated hepatocellular carcinoma. Am J Surg Pathol 2005;29:1600-8.

8. Wee A. Diagnostic utility of immunohistochemistry in hepatocellular carcinoma, its variants and their mimics. Appl Immunohistochem Mol Morphol 2006;14:266-72.

9. Marrero JA. Modern diagnosis of hepatocellular carcinoma: utilization of liver biopsy and genomic markers. J Hepatol 2009;50:659-61.

10. Cao R, Wang L, Wang H, et al. Role of histone H3 lysine 27 methylation in polycombgroup silencing. Science 2002;298:1039-43.

11. Simon JA, Lange CA. Roles of the EZH2 histone methyltransferase in cancer epigenetics. Mutat Res 2008;647:21-9.

12. Varambally $\mathbf{S}$, Dhanasekaran SM, Zhou M, et al. The polycomb group protein EZH2 is involved in progression of prostate cancer. Nature 2002;419:624-9.

13. Chen $\mathbf{Y}$, Lin MC, Yao $\mathrm{H}$, et al. Lentivirus-mediated RNA interference targeting enhancer of zeste homolog 2 inhibits hepatocellular carcinoma growth through downregulation of stathmin. Hepatology 2007:46:200-8.

14. Sudo T, Utsunomiya T, Mimori K, et al. Clinicopathological significance of EZH2 mRNA expression in patients with hepatocellular carcinoma. $\mathrm{Br} \mathrm{J}$ Cancer 2005;92:1754-8.

15. Yonemitsu Y, Imazeki F, Chiba T, et al. Distinct expression of polycomb group proteins EZH2 and BMI1 in hepatocellular carcinoma. Hum Pathol 2009;40:1304-11.

16. Sasaki M, Ikeda $\mathrm{H}$, Itatsu K, et al. The overexpression of polycomb group proteins Bmi1 and EZH2 is associated with the progression and aggressive biological behavior of hepatocellular carcinoma. Lab Invest 2008;88:873-82.

17. Capurro M, Wanless IR, Sherman M, et al. Glypican-3: a novel serum and histochemical marker for hepatocellular carcinoma. Gastroenterology 2003;125:89-97.

18. Yamauchi N, Watanabe A, Hishinuma $\mathrm{M}$, et al. The glypican 3 oncofetal protein is a promising diagnostic marker for hepatocellular carcinoma. Mod Pathol 2005:18:1591-8

19. Llovet JM, Chen $\mathrm{Y}$, Wurmbach E, et al. A molecular signature to discriminate dysplastic nodules from early hepatocellular carcinoma in HCV cirrhosis. Gastroenterology 2006;131:1758-67.

20. Chuma M, Sakamoto M, Yamazaki K, et al. Expression profiling in multistage hepatocarcinogenesis: identification of HSP70 as a molecular marker of early hepatocellular carcinoma. Hepatology 2003;37:198-207.

21. Di Tommaso L, Franchi G, Park YN, et al. Diagnostic value of HSP70, glypican 3, and glutamine synthetase in hepatocellular nodules in cirrhosis. Hepatology 2007:45:725-34.

22. Di Tommaso L, Destro A, Seok JY, et al. The application of markers (HSP70, GPC3 and GS) in liver biopsies is useful for detection of hepatocellular carcinoma. $J$ Hepatol 2009;50:746-54.

23. Terminology of nodular hepatocellular lesions. International Working Party. Hepatology 1995;22:983-93.

24. Sobin LH, Fleming ID. TNM Classification of Malignant Tumors, fifth edition (1997) Union Internationale Contre le Cancer and the American Joint Committee on Cancer. Cancer 1997;80:1803-4.

25. Xie D, Sham JS, Zeng WF, et al. Heterogeneous expression and association of betacatenin, p16 and c-myc in multistage colorectal tumorigenesis and progression detected by tissue microarray. Int J Cancer 2003;107:896-902. 
26. Cai MY, Zhang B, He WP, et al. Decreased expression of PinX1 protein is correlated with tumor development and is a new independent poor prognostic factor in ovarian carcinoma. Cancer Sci 2010;101:1543-9.

27. Seimiya $\mathbf{M}$, Tomonaga $T$, Matsushita $K$, et al. Identification of nove immunohistochemical tumor markers for primary hepatocellular carcinoma; clathrin heavy chain and formiminotransferase cyclodeaminase. Hepatology 2008:48:519-30.

28. Forner A, Vilana R, Ayuso C, et al. Diagnosis of hepatic nodules $20 \mathrm{~mm}$ or smaller in cirrhosis: prospective validation of the noninvasive diagnostic criteria for hepatocellular carcinoma. Hepatology 2008:47:97-104.
29. Forner A, Bruix J. Locoregional treatment for hepatocellular carcinoma: from clinical exploration to robust clinical data, changing standards of care. Hepatology 2008;47:5-7.

30. Kojiro M, Roskams T. Early hepatocellular carcinoma and dysplastic nodules. Semin Liver Dis 2005:25:133-42.

31. Roncalli M. Hepatocellular nodules in cirrhosis: focus on diagnostic criteria on live biopsy. A Western experience. Liver Transp/ 2004:10:S9-15.

32. Roncalli M, Borzio M, Di Tommaso L. Hepatocellular dysplastic nodules. Hepatol Res 2007;37(Suppl 2):S125-34.

33. Hytiroglou P, Park YN, Krinsky G, et al. Hepatic precancerous lesions and small hepatocellular carcinoma. Gastroenterol Clin North Am 2007;36:867-87, vii.

\section{Editor's quiz: GI snapshot}

\section{ANSWER}

From the question on page 922

The diagnosis was acute pancreatitis complicated with pseudo-cyst and pseudo-aneurysm of splenic artery.

The colour Doppler and three-dimensional sonogram revealed the nodule to be hyper-vascular and communicating with the splenic artery (figures 1 and 2). A CT scan (figure 3) and its volume rendering reformatted angiography (figure 4) showed a pseudo-cyst and a pseudo-aneurysm from the splenic artery. Therefore, acute pancreatitis complicated with a pseudo-cyst and pseudo-aneurysm was diagnosed. The pseudo-aneurysm was removed by surgery uneventfully and minimal rupture was disclosed during the operation.

Rupture of a pseudo-aneurysm is a rare complication of pancreatitis, and is associated with poor prognosis. The mortality correlates with the severity of the pseudo-aneurysm and the overall mortality of acute pancreatitis, around $7.8 \%$. The pseudo-aneurysm caused by acute pancreatitis usually develops

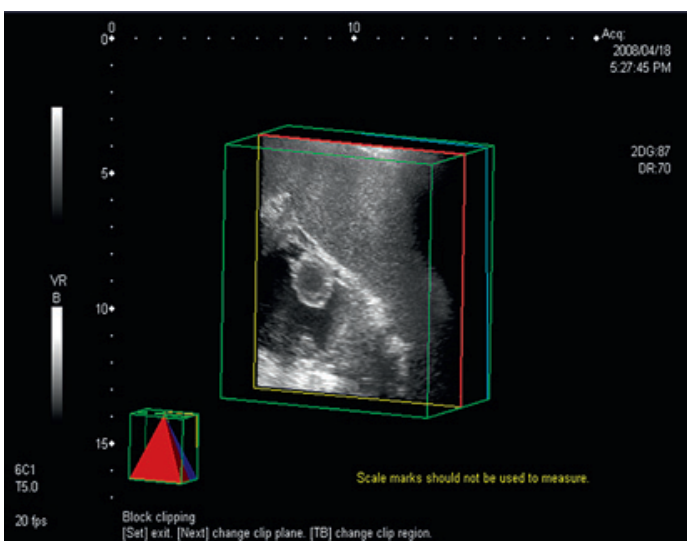

Figure 1 Three-dimensional sonogram.

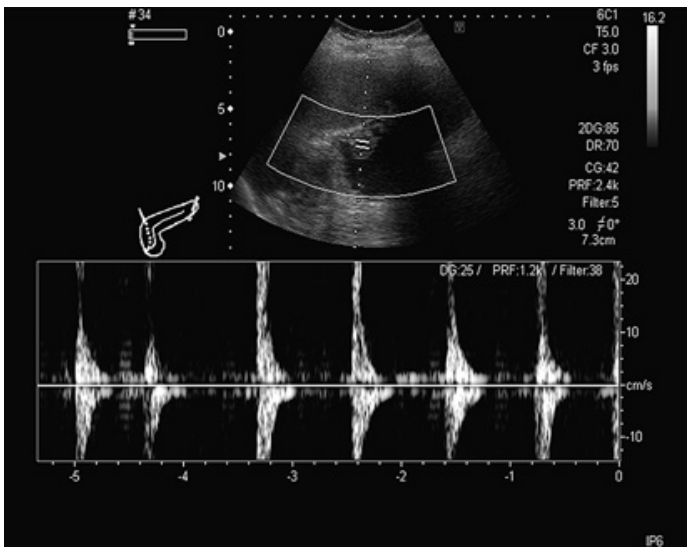

Figure 2 Color Doppler sonogram.

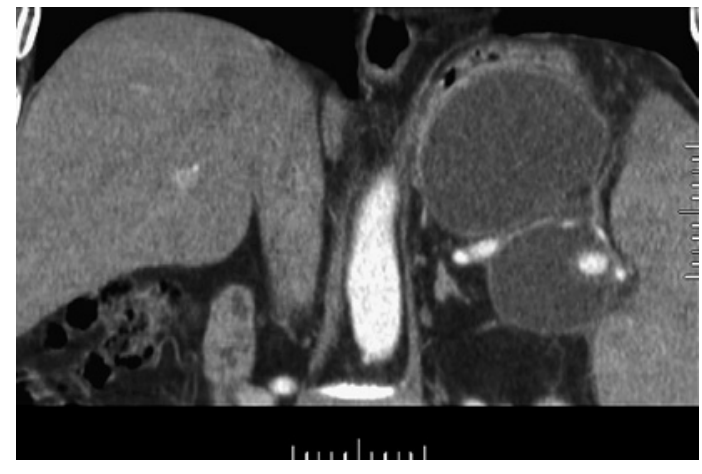

Figure 3 Pseudo-aneurysm from the splenic artery by CT scan.

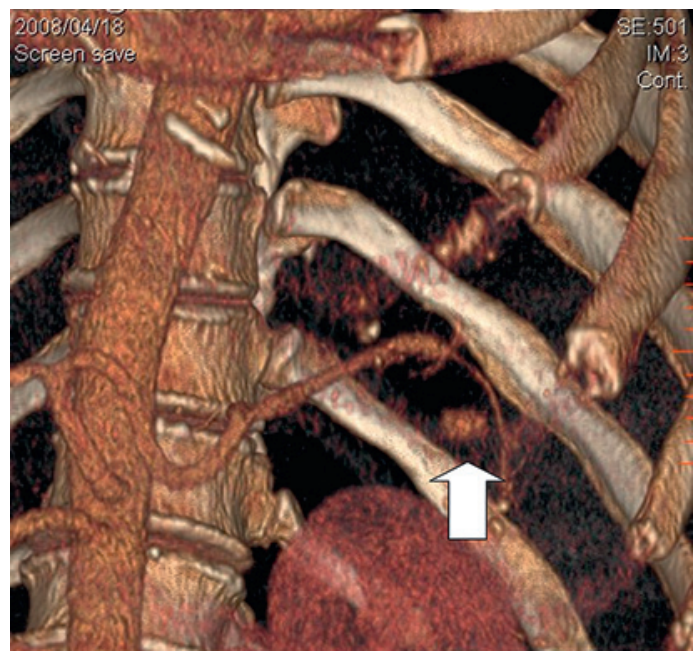

Figure 4 Reformatted angiography. The arrow indicates the pseudoaneurysm.

at superior mesentery artery, splenic artery or gastro-duodenal artery with manifestation of gastro-intestine bleeding, haemobilia, obstructive jaundice, or internal bleeding. Angiography is usually required for confirmation of the diagnosis. Non-invasive imaging techniques such as colour Doppler ultrasound and contrast-enhanced CT scan are also useful in diagnosing pancreatic pseudo-aneurysm associated with acute pancreatitis. ${ }^{1-3}$

Gut 2011;60:976. doi:10.1136/gut.2010.211854a

\section{REFERENCES}

1. Jabłoński S, Brocki M, Sapiezko J, et al. The results of treatment of acute haemorrhagic necrotizing pancreatitis in the own material. Pol Merkur Lekarski 2004;17(Suppl 1):156-9.

2. Bergert $\mathbf{H}$, Hinterseher I, Kersting S, et al. Management and outcome of hemorrhage due to arterial pseudoaneurysms in pancreatitis. Surgery 2005;137:323-8.

3. Zhou F, Wang C, Xiong J, et al. Experience in diagnosis and treatment of bleeding complications in severe acute pancreatitis by TAE. J Huazhong Univ Sci Technolog Med Sci 2005;25:182-4 


\section{GUT}

\section{EZH2 protein: a promising immunomarker for the detection of hepatocellular carcinomas in liver needle biopsies}

Mu-Yan Cai, Zhu-Ting Tong, Fang Zheng, et al.

Gut 2011 60: 967-976 originally published online February 17, 2011 doi: $10.1136 /$ gut.2010.231993

Updated information and services can be found at:

http://gut.bmj.com/content/60/7/967.full.html

These include:

References This article cites 33 articles, 2 of which can be accessed free at: http://gut.bmj.com/content/60/7/967.full.html\#ref-list-1

Article cited in:

http://gut.bmj.com/content/60/7/967.full.html\#related-urls

Email alerting Receive free email alerts when new articles cite this article. Sign up in service the box at the top right corner of the online article.

Topic Articles on similar topics can be found in the following collections Collections

$$
\text { Hepatic cancer (337 articles) }
$$

Notes

To request permissions go to:

http://group.bmj.com/group/rights-licensing/permissions

To order reprints go to:

http://journals.bmj.com/cgi/reprintform

To subscribe to BMJ go to:

http://group.bmj.com/subscribe/ 